TAMKANG JOURNAL OF MATHEMATICS

Volume 41, Number 4, 325-333, Winter 2010

\title{
FIBONACCI-TRIPLE SEQUENCES AND SOME FUNDAMENTAL PROPERTIES
}

\author{
BIJENDRA SINGH AND OMPRAKASH SIKHWAL
}

\begin{abstract}
Fibonacci sequence stands as a kind of super sequence with fabulous properties. This note presents Fibonacci-Triple sequences that may also be called 3F sequences. This is the explosive development in the region of Fibonacci sequence. Our purpose of this paper is to demonstrate fundamental properties of FibonacciTriple sequence.
\end{abstract}

\section{Introduction}

The Fibonacci-Triple sequence is a new direction in generalization of coupled Fibonacci sequences. Much work has been done to study on Fibonacci-Triple sequence. The concept of Fibonacci-Triple sequence was first introduced by J. Z. Lee and J. S. Lee [1]. He described specific scheme and derived recurrent formula. K. T. Atanassov [2], [3] described new ideas for Fibonacci-Triple sequences and called 3-Fibonacci sequences or 3-F sequences. The Fibonacci-Triple sequence involves three sequences of integers in which the elements of one sequence are part of the generalization of the other, and vice versa.

In this paper we present basic concepts that will be used to construct Fibonacci-Triple sequences of second order with fascinating properties. At present, we shall describe two specific schemes for 3-F sequence and fundamental properties with proof.

\section{Fibonacci-triple sequence of second order}

Fibonacci-Triple sequence is the explosive development in the region of Fibonacci sequence. Let $\left\{\alpha_{i}\right\}_{i=0}^{\infty},\left\{\beta_{i}\right\}_{i=0}^{\infty}$, and $\left\{\gamma_{i}\right\}_{i=0}^{\infty}$, be three infinite sequences and six arbitrary real numbers $a, b, c, d, e$ and $f$ be given. Then J. Z. Lee and J. S. Lee [1] defined following specific scheme for three sequences and derived recurrent formula:

Corresponding author: Omprakash Sikhwal.

Received September 16, 2009.

2000 Mathematics Subject Classification. 11B39, 11 B37. 


\section{First Scheme:}

$$
\begin{array}{ll}
\alpha_{0}=a, \beta_{0}=b, \gamma_{0}=c, \alpha_{1}=d, \beta_{1}=e, \gamma_{1}=f \\
\alpha_{n+2}=\beta_{n+1}+\gamma_{n}, & n \geq 0 \\
\beta_{n+2}=\gamma_{n+1}+\alpha_{n}, & n \geq 0 \\
\gamma_{n+2}=\alpha_{n+1}+\beta_{n}, & n \geq 0
\end{array}
$$

K. Atanassov [3] considers the following specific scheme:

\section{Second Scheme:}

$$
\begin{array}{ll}
\alpha_{0}=a, \beta_{0}=b, \gamma_{0}=c, \alpha_{1}=d, \beta_{1}=e, \gamma_{1}=f \\
\alpha_{n+2}=\gamma_{n+1}+\beta_{n}, & n \geq 0 \\
\beta_{n+2}=\alpha_{n+1}+\alpha_{n}, & n \geq 0 \\
\gamma_{n+2}=\beta_{n+1}+\alpha_{n}, & n \geq 0 .
\end{array}
$$

K. T. Atanassov [2], [3] notified 36 different schemes of 3-F sequences. There are ten schemes are trivial 3-F sequences because they having at least one resulting sequence same as Fibonacci sequence. The twenty six remaining schemes are essential generalization of the Fibonacci sequence. These sequences have arranged in seven groups and called as basic 3-F sequences.

\section{Properties of first scheme}

First few terms of First scheme (1) are as under:

\begin{tabular}{|c|c|c|c|}
\hline$n$ & $\alpha_{n}$ & $\beta_{n}$ & $\gamma_{n}$ \\
\hline 0 & $a$ & $b$ & $c$ \\
\hline 1 & $d$ & $e$ & $f$ \\
\hline 2 & $c+e$ & $a+f$ & $b+d$ \\
\hline 3 & $a+2 f$ & $b+2 d$ & $c+2 e$ \\
\hline 4 & $2 b+3 d$ & $2 c+3 e$ & $2 a+3 f$ \\
\hline 5 & $3 c+5 e$ & $3 a+5 f$ & $3 b+5 d$ \\
\hline 6 & $5 a+8 f$ & $5 b+8 d$ & $5 c+8 e$ \\
\hline 7 & $8 b+13 d$ & $8 c+13 e$ & $8 a+13 f$ \\
\hline 8 & $13 c+21 e$ & $13 a+21 f$ & $13 b+21 d$ \\
\hline 9 & $21 a+34 f$ & $21 b+34 d$ & $21 c+34 e$ \\
\hline 10 & $34 b+55 d$ & $34 c+55 e$ & $34 a+55 f$ \\
\hline
\end{tabular}

If we set $a=b=c$ and $d=e=f$, then the sequences $\left\{\alpha_{i}\right\}_{i=0}^{\infty},\left\{\beta_{i}\right\}_{i=0}^{\infty}$ and $\left\{\gamma_{i}\right\}_{i=0}^{\infty}$. Will be coincide with each other and with the sequence $\left\{F_{i}\right\}_{i=0}^{\infty}$, which is called a generalized 
Fibonacci sequence, where

$$
F_{0}(a, d)=a, F_{1}(a, d)=d, F_{n+2}(a, d)=F_{n+1}(a, d)+F_{n}(a, d) .
$$

Now we present fundamental properties of First scheme.

Theorem 1. For every integer $n \geq 0$,

(a) $\alpha_{n+6}=4 \alpha_{n+3}+\alpha_{n}$,

(b) $\beta_{n+6}=4 \beta_{n+3}+\beta_{n}$,

(c) $\gamma_{n+6}=4 \gamma_{n+3}+\gamma_{n}$.

Proof. (a) To prove this, we shall use induction method. If $n=0$ then

$$
\begin{aligned}
\alpha_{6} & =\beta_{5}+\gamma_{4}=\gamma_{4}+\alpha_{3}+\gamma_{4}=2 \gamma_{4}+\alpha_{3} & & \text { (by First scheme 1) } \\
& =2 \alpha_{3}+2 \beta_{2}+\alpha_{3}=3 \alpha_{3}+\beta_{2}+\gamma_{1}+\alpha_{0} & & \text { (by First scheme 1) } \\
& =3 \alpha_{3}+\alpha_{3}+\alpha_{0} & & \text { (by First scheme 1) } \\
& =4 \alpha_{3}+\alpha_{0} & &
\end{aligned}
$$

Thus the result is true for $n=0$.

Let us assume that the result is true for some integer $n \geq 1$. then

$$
\begin{array}{rlrl}
\alpha_{n+7} & =\beta_{n+6}+\gamma_{n+5} & \\
& =\gamma_{n+5}+\alpha_{n+4}+\alpha_{n+4}+\beta_{n+3}=\gamma_{n+5}+2 \alpha_{n+4}+\beta_{n+3} & & \text { (by First scheme) } \\
& =\alpha_{n+4}+\beta_{n+3}+2 \alpha_{n+4}+\beta_{n+3}=3 \alpha_{n+4}+2 \beta_{n+3} & & \text { (by First scheme) } \\
& =3 \alpha_{n+4}+2 \gamma_{n+2}+2 \alpha_{n+1}=3 \alpha_{n+4}+\gamma_{n+2}+\alpha_{n+1}+\beta_{n}+2 \alpha_{n+1} & \text { (by First scheme) } \\
& =3 \alpha_{n+4}+\beta_{n+3}+\beta_{n}+2 \alpha_{n+1} & & \text { (by First scheme) } \\
& =3 \alpha_{n+4}+\beta_{n+3}+\gamma_{n+2}+\alpha_{n+1} & & \text { (by First scheme) } \\
& =4 \alpha_{n+4}+\alpha_{n+1} & & \text { (by First scheme) }
\end{array}
$$

Hence the result is true for all integers $n \geq 0$.

Similar proofs can be given for remaining parts (b) and (c).

Theorem 2. For every integer $n \geq 0$,

(a) $\alpha_{n}=\left\{\begin{array}{l}F_{n-1} a+F_{n} f, \text { if } n \equiv 0(\bmod 3) \\ F_{n-1} b+F_{n} d, \text { if } n \equiv 1(\bmod 3), \\ F_{n-1} c+F_{n} e, \text { if } n \equiv 2(\bmod 3)\end{array}\right.$
(b) $\beta_{n}=\left\{\begin{array}{l}F_{n-1} b+F_{n} d, \text { if } n \equiv 0(\bmod 3) \\ F_{n-1} c+F_{n} e, \text { if } n \equiv 1(\bmod 3), \\ F_{n-1} a+F_{n} f, \text { if } n \equiv 2(\bmod 3)\end{array}\right.$ 
(c) $\alpha_{n}=\left\{\begin{array}{l}F_{n-1} c+F_{n} e, \text { if } n \equiv 0(\bmod 3) \\ F_{n-1} a+F_{n} f, \text { if } n \equiv 1(\bmod 3) . \\ F_{n-1} b+F_{n} d, \text { if } n \equiv 2(\bmod 3)\end{array}\right.$

Proof. (a) By Theorem (1), we have

$$
\alpha_{n+6}=4 \alpha_{n+3}+\alpha_{n}, \quad \text { for } n \geq 0 .
$$

When $n \equiv 0(\bmod 3)$, assume $n=3 m$, we have

$$
\alpha_{3(m+2)}=4 \alpha_{3(m+1)}+\alpha_{3 m}, \quad \text { with } \alpha_{0}=a, \alpha_{3}=a+2 f .
$$

Let $G_{m}=\alpha_{3 m}$, we have

$$
G_{m+2}=4 G_{m+1}+G_{m}, \quad \text { with } G_{0}=a, G_{1}=a+2 f
$$

Therefore, we get

$$
\begin{aligned}
G_{m} & =\frac{(\sqrt{5}-1) a+2 f}{2 \sqrt{5}}(2+\sqrt{5})^{m}+\frac{(\sqrt{5}-1) a-2 f}{2 \sqrt{5}}(2-\sqrt{5})^{m} \\
& =\frac{1}{\sqrt{5}}\left[a\left\{\left(\frac{1+\sqrt{5}}{2}\right)^{3 m-1}-\left(\frac{1-\sqrt{5}}{2}\right)^{3 m-1}\right\}+f\left\{\left(\frac{1+\sqrt{5}}{2}\right)^{3 m}-\left(\frac{1-\sqrt{5}}{2}\right)^{3 m}\right\}\right] \\
& =F_{n-1} a+F_{n} f
\end{aligned}
$$

i.e., $\alpha_{n}=F_{n-1} a+F_{n} f$.

Using same process to get: $\alpha_{n}=\left\{\begin{array}{l}F_{n-1} a+F_{n} f, \text { if } n \equiv 0(\bmod 3) \\ F_{n-1} b+F_{n} d, \text { if } n \equiv 1(\bmod 3), \\ F_{n-1} c+F_{n} e, \text { if } n \equiv 2(\bmod 3)\end{array}\right.$

Similar proofs can be given for remaining parts (b) and (c).

Theorem 3. For every integer $n \geq 0$,

$$
\alpha_{n}+\beta_{n}+\gamma_{n}=F_{n-1}\left(\alpha_{0}+\beta_{0}+\gamma_{0}\right)+F_{n}\left(\alpha_{1}+\beta_{1}+\gamma_{1}\right) .
$$

Proof follows from Theorem 2.

Theorem 4. For every integer $n \geq 0$,

$$
\sum_{k=0}^{n}\left(\alpha_{k}+\beta_{k}+\gamma_{k}\right)=F_{n+1}\left(\alpha_{0}+\beta_{0}+\gamma_{0}\right)+\left(F_{n+2}-1\right)\left(\alpha_{1}+\beta_{1}+\gamma_{1}\right) .
$$


Proof. (a) To prove this, we shall use induction method.

If $n=0$ the result is obviously true, since

$$
\alpha_{0}+\beta_{0}+\gamma_{0}=F_{1}\left(a_{0}+\beta_{0}+\gamma_{0}\right)+\left(F_{2}-1\right)\left(\alpha_{1}+\beta_{1}+\gamma_{1}\right) .
$$

Let us assume that the result is true for some integer $n \geq 1$, then

$$
\begin{aligned}
& \sum_{k=0}^{n+1}\left(\alpha_{k}+\beta_{k}+\gamma_{k}\right) \\
& =\left(\alpha_{n+1}+\beta_{n+1}+\gamma_{n+1}\right)+\sum_{k=0}^{n}\left(\alpha_{k}+\beta_{k}+\gamma_{k}\right) \\
& =\left(\alpha_{n+1}+\beta_{n+1}+\gamma_{n+1}\right)+F_{n+1}\left(\alpha_{0}+\beta_{0}+\gamma_{0}\right)+\left(F_{n+2}-1\right)\left(\alpha_{1}+\beta_{1}+\gamma_{1}\right) \\
& =F_{n}\left(\alpha_{0}+\beta_{0}+\gamma_{0}\right)+F_{n+1}\left(\alpha_{1}+\beta_{1}+\gamma_{1}\right)+F_{n+1}\left(\alpha_{0}+\beta_{0}+\gamma_{0}\right) \\
& \quad+\left(F_{n+2}-1\right)\left(\alpha_{1}+\beta_{1}+\gamma_{1}\right) \\
& =F_{n+2}\left(\alpha_{0}+\beta_{0}+\gamma_{0}\right)+\left(F_{n+3}-1\right)\left(\alpha_{1}+\beta_{1}+\gamma_{1}\right)
\end{aligned}
$$

Hence the result is true for all integers $n \geq 0$.

Theorem 5. For every integer $n \geq 0$,

$$
\left(\alpha_{n+2}+\beta_{n+2}+\gamma_{n+2}\right)=\sum_{k=0}^{n}\left(\alpha_{k}+\beta_{k}+\gamma_{k}\right)+\left(\alpha_{1}+\beta_{1}+\gamma_{1}\right) .
$$

Proof can be given by induction method.

Theorem 6. For every integer $n \geq 0$,

(a) $\alpha_{3 n+6}-2 \alpha_{3 n+3}=\alpha_{0} L_{3 n+2}+\gamma_{1} L_{3 n+3}$,

(b) $\alpha_{3 n+7}-2 \alpha_{3 n+4}=\beta_{0} L_{3 n+3}+\alpha_{1} L_{3 n+4}$,

(c) $\alpha_{3 n+8}-2 \alpha_{3 n+5}=\gamma_{0} L_{3 n+4}+\beta_{1} L_{3 n+5}$,

(d) $\beta_{3 n+6}-2 \beta_{3 n+3}=\beta_{0} L_{3 n+2}+\alpha_{1} L_{3 n+3}$,

(e) $\beta_{3 n+7}-2 \beta_{3 n+4}=\gamma_{0} L_{3 n+3}+\beta_{1} L_{3 n+4}$,

(f) $\beta_{3 n+8}-2 \beta_{3 n+5}=\alpha_{0} L_{3 n+4}+\gamma_{1} L_{3 n+5}$,

(g) $\gamma_{3 n+6}-2 \gamma_{3 n+3}=\gamma_{0} L_{3 n+2}+\beta_{1} L_{3 n+3}$,

(h) $\gamma_{3 n+7}-2 \gamma_{3 n+4}=\alpha_{0} L_{3 n+3}+\gamma_{1} L_{3 n+4}$,

(i) $\gamma_{3 n+8}-2 \gamma_{3 n+5}=\beta_{0} L_{3 n+4}+\alpha_{1} L_{3 n+5}$.

Proof. (a) We know that by Theorem 2,

$$
\alpha_{3 n+3}=\alpha_{0} F_{3 n+2}+\gamma_{1} F_{3 n+3} .
$$


Also

$$
L_{3 n+5}=2 F_{3 n+5}+F_{3 n+2} \text { and } L_{3 n+6}=2 L_{3 n+6}+F_{3 n+3} .
$$

If $n=0$ then

$$
\begin{array}{rlrl}
\alpha_{6}-2 \alpha_{3} & =4 \alpha_{3}+\alpha_{0}-2 \alpha_{3} & & \text { (by Theorem 1) } \\
& =2 \alpha_{3}+\alpha_{0} & & \\
& =2 F_{2} \alpha_{0}+2 F_{3} \gamma_{1}+\alpha_{0} & & \text { (by equation (5) } \\
& =\left(2 F_{2}+1\right) \alpha_{0}+2 F_{3} \gamma_{1} & & \\
& =L_{2} \alpha_{0}+L_{3} \gamma_{1} . &
\end{array}
$$

Thus the result is true for $n=0$.

Let us assume that the result is true for some integer $n \geq 1$, then

$$
\begin{aligned}
& \alpha_{3 n=9}-2 \alpha_{3 n+6}=4 \alpha_{3 n+6}+\alpha_{3 n+3}-2 \alpha_{3 n+6} \quad \text { (by Theorem 1) } \\
& =2 \alpha_{3 n+6}+\alpha_{3 n+3} \\
& =2\left(F_{3 n+5} \alpha_{0}+F_{3 n+6} \gamma_{1}\right)+\left(F_{3 n+2} \alpha_{0}+F_{3 n+3} \gamma_{1}\right) \quad \text { (by Equation (5)) } \\
& =\left(2 F_{3 n+5}+F_{3 n+2}\right) \alpha_{0}+\left(2 F_{3 n+6}+F_{3 n+3}\right) \gamma_{1} \\
& =\alpha_{0} L_{3 n+5}+\gamma_{1} L_{3 n+6} \\
& \text { (by Equation (6)) }
\end{aligned}
$$

Hence the result is true for all integers $n \geq 0$.

Similar proofs can be given for remaining parts (b) to (i).

Theorem 7. For every integer $n \geq 0$,

(a) $\sum_{k=0}^{n}\left(\gamma_{3 k+4}+\alpha_{3 k+3}\right)=\sum_{k=0}^{n} \beta_{3 k+5}$,

(b) $\sum_{k=0}^{n}\left(\alpha_{3 k+4}+\beta_{3 k+3}\right)=\sum_{k=0}^{n} \gamma_{3 k+5}$,

(c) $\sum_{k=0}^{n}\left(\beta_{3 k+4}+\gamma_{3 k+3}\right)=\sum_{k=0}^{n} \alpha_{3 k+5}$,

Proofs can be given by induction method.

Now we present fundamental properties of second scheme (2).

\section{Properties Of Second Scheme}

First few terms of second scheme (2) are as under: 


\begin{tabular}{|c|c|c|c|}
\hline$n$ & $\alpha_{n}$ & $\beta_{n}$ & $\gamma_{n}$ \\
\hline 0 & $a$ & $b$ & $c$ \\
\hline 1 & $d$ & $e$ & $f$ \\
\hline 2 & $b+f$ & $c+d$ & $a+e$ \\
\hline 3 & $a+2 e$ & $b+2 f$ & $c+2 d$ \\
\hline 4 & $2 c+3 d$ & $2 a+3 e$ & $2 b+3 f$ \\
\hline 5 & $3 b+5 f$ & $3 c+5 d$ & $3 a+5 e$ \\
\hline 6 & $5 a+8 e$ & $5 b+8 f$ & $5 c+8 d$ \\
\hline 7 & $8 c+13 d$ & $8 a+13 e$ & $8 b+13 f$ \\
\hline 8 & $13 b+21 f$ & $13 c+21 d$ & $13 a+21 e$ \\
\hline 9 & $21 a+34 e$ & $21 b+34 f$ & $21 c+34 d$ \\
\hline 10 & $34 b c+55 d$ & $34 a+55 e$ & $34 b+55 f$ \\
\hline
\end{tabular}

Theorem 8. For every integer $n \geq 0$,

(a) $\alpha_{n+6}=4 \alpha_{n+3}+\alpha_{n}$,

(b) $\beta_{n+6}=4 \beta_{n+3}+\beta_{n}$,

(c) $\gamma_{n+6}=4 \gamma_{n+3}+\gamma_{n}$.

Theorem 9. For every integer $n \geq 0$,

(a) $\alpha_{n}=\left\{\begin{array}{l}F_{n-1} a+F_{n} e, \text { if } n \equiv 0(\bmod 3) \\ F_{n-1} c+F_{n} d, \text { if } n \equiv 1(\bmod 3), \\ F_{n-1} b+F_{n} f, \text { if } n \equiv 2(\bmod 3)\end{array}\right.$

(b) $\beta_{n}=\left\{\begin{array}{l}F_{n-1} b+F_{n} f, \text { if } n \equiv 0(\bmod 3) \\ F_{n-1} a+F_{n} e, \text { if } n \equiv 1(\bmod 3), \\ F_{n-1} c+F_{n} d, \text { if } n \equiv 2(\bmod 3)\end{array}\right.$

(c) $\alpha_{n}=\left\{\begin{array}{l}F_{n-1} c+F_{n} d, \text { if } n \equiv 0(\bmod 3) \\ F_{n-1} b+F_{n} f, \text { if } n \equiv 1(\bmod 3) . \\ F_{n-1} a+F_{n} e, \text { if } n \equiv 2(\bmod 3)\end{array}\right.$

Theorem 10. For every integer $n \geq 0$,

$$
\alpha_{n}+\beta_{n}+\gamma_{n}=F_{n-1}\left(\alpha_{0}+\beta_{0}+\gamma_{0}\right)+F_{n}\left(\alpha_{1}+\beta_{1}+\gamma_{1}\right)
$$

Theorem 11. For every integer $n \geq 0$,

$$
\sum_{k=0}^{n}\left(\alpha_{k}+\beta_{k}+\gamma_{k}\right)=F_{n+1}\left(\alpha_{0}+\beta_{0}+\gamma_{0}\right)+\left(F_{n+2}-1\right)\left(\alpha_{1}+\beta_{1}+\gamma_{1}\right)
$$

Theorem 12. For every integer $n \geq 0$,

$$
\left(\alpha_{n+2}+\beta_{n+2}+\gamma_{n+2}\right)=\sum_{k=0}^{n}\left(\alpha_{k}+\beta_{k}+\gamma_{k}\right)+\left(\alpha_{1}+\beta_{1}+\gamma_{1}\right)
$$


Theorem 13. For every integer $n \geq 0$,

(a) $\alpha_{3 n+6}-2 \alpha_{3 n+3}=\alpha_{0} L_{3 n+2}+\beta_{1} L_{3 n+3}$,

(b) $\alpha_{3 n+7}-2 \alpha_{3 n+4}=\gamma_{0} L_{3 n+3}+\alpha_{1} L_{3 n+4}$,

(c) $\alpha_{3 n+8}-2 \alpha_{3 n+5}=\beta_{0} L_{3 n+4}+\gamma_{1} L_{3 n+5}$,

(d) $\beta_{3 n+6}-2 \beta_{3 n+3}=\beta_{0} L_{3 n+2}+\gamma_{1} L_{3 n+3}$,

(e) $\beta_{3 n+7}-2 \beta_{3 n+4}=\alpha_{0} L_{3 n+3}+\beta_{1} L_{3 n+4}$,

(f) $\beta_{3 n+8}-2 \beta_{3 n+5}=\gamma_{0} L_{3 n+4}+\alpha_{1} L_{3 n+5}$,

(g) $\gamma_{3 n+6}-2 \gamma_{3 n+3}=\gamma_{0} L_{3 n+2}+\alpha_{1} L_{3 n+3}$,

(h) $\gamma_{3 n+7}-2 \gamma_{3 n+4}=\beta_{0} L_{3 n+3}+\gamma_{1} L_{3 n+4}$,

(i) $\gamma_{3 n+8}-2 \gamma_{3 n+5}=\alpha_{0} L_{3 n+4}+\beta_{1} L_{3 n+5}$.

Theorem 14. For every integer $n \geq 0$,

(a) $\sum_{k=0}^{n}\left(\alpha_{3 k+3}+\beta_{3 k+4}\right)=\sum_{k=0}^{n} \gamma_{3 k+5}$,

(b) $\sum_{k=0}^{n}\left(\gamma_{3 k+3}+\alpha_{3 k+4}\right)=\sum_{k=0}^{n} \beta_{3 k+5}$,

(c) $\sum_{k=0}^{n}\left(\beta_{3 k+3}+\gamma_{3 k+4}\right)=\sum_{k=0}^{n} \alpha_{3 k+5}$.

The proofs of the above fundamental properties of second scheme (2) are similar to those in first scheme (1).

\section{Conclusion}

This paper describes Fibonacci-Triple sequences of second order. We have stated and derived many fundamental properties in this paper. The concept of such generalization can be implemented over classical recurrent sequences. The idea can be extended for higher order Fibonacci-Triple sequences and similar properties can be produced in case of higher order Fibonacci-Triple sequences. By varying the pattern, it is easy to establish surprising results in lucid manner.

\section{References}

[1] J. Z. Lee and J. S. Lee, Some properties of the generalization of the Fibonacci sequence, The Fibonacci Quarterly, 25 (1987), 111-117.

[2] K. Atanassov, On a generalization of the Fibonacci sequence in the case of three sequences, The Fibonacci Quarterly 27 (1989), 7-10.

[3] K. Atanassov, V. Atanassov, A. Shannon and J. Turner. New Visual Perspectives On Fibonacci Numbers, World Scientific, 2002. 
[4] N. Vorobyov, The Fibonacci Numbers, D. C. Health and Company, Boston, 1963.

School of Studies in Mathematics, Vikram University Ujjain, India.

E-mail: bijendrasingh@yahoo.com

Department of Mathematics, Mandsaur Institute of Technology,Mandsaur, India.

E-mail: opbhsikhwal@rediffmail.com 